\title{
Viremic long-term nonprogressive HIV-1 infection is not associated with abnormalities in known Nef functions
}

\author{
Anke Heigele ${ }^{1}$, David Camerini ${ }^{2}$, Angélique B van't Wout ${ }^{3,4}$ and Frank Kirchhoff ${ }^{*}$
}

\begin{abstract}
Background: A small minority of HIV-1-infected individuals show low levels of immune activation and do not develop immunodeficiency despite high viral loads. Since the accessory viral Nef protein modulates T cell activation and plays a key role in the pathogenesis of AIDS, we investigated whether specific properties of Nef may be associated with this highly unusual clinical outcome of HIV-1 infection.

Findings: Comprehensive functional analyses of sequential HIV-1 strains from three viremic long-term non-progressors (VNP) showed that they encode full-length Nef proteins that are capable of modulating CD4, CD28, CD8ß, MHC-I and CD74 cell surface expression. Similar to Nef proteins from HIV-1-infected individuals with progressive infection (P-Nefs) and unlike Nefs from simian immunodeficiency viruses (SIVs) that do not cause chronic immune activation and disease in their natural simian hosts, VNP-Nefs were generally unable to down-modulate TCR-CD3 cell surface expression to block T cell activation and apoptosis. On average, VNP-Nefs suppressed NF-AT activation less effectively than P-Nefs and were slightly less active in enhancing NF-KB activity. Finally, we found that VNP-Nefs increased virion infectivity and enhanced HIV-1 replication and cytopathicity in primary human cells and in ex vivo infected lymphoid tissues.

Conclusions: Our results show that nef alleles from VNPs and progressors of HIV-1 infection show only modest differences in established functions. Thus, the lack of chronic immune activation and disease progression in HIV1-infected VNPs is apparently not associated with unusual functional properties of the accessory viral Nef protein.
\end{abstract}

Keywords: HIV-1, Viremic long-term non-progressors, Immune activation, Nef function

\section{Findings}

High viral loads are almost invariably associated with chronic inflammation and progression to AIDS in HIV1-infected individuals. In contrast, some non-human primates (NHPs) that are naturally infected with SIVs, such as sooty mangabeys (SMs) or African green monkeys (AGMs), show low levels of immune activation and do not develop disease despite high levels of viral replication $[1,2]$. Recent data show that a small minority $(<1 \%)$ of highly viremic HIV-1-infected individuals, so called viremic long-term non-progressors (VNP), show a similar phenotype and remain asymptomatic with low levels of inflammation and high $\mathrm{CD} 4^{+} \mathrm{T}$ cell counts [3].

\footnotetext{
* Correspondence: frank.kirchhoff@uni-ulm.de

${ }^{1}$ Institute of Molecular Virology, Ulm University Medical Center, Ulm, Germany

Full list of author information is available at the end of the article
}

It is poorly understood why VNPs can tolerate high levels of HIV-1 replication. It has been reported, however, that they show lower levels of proliferating and activated $T$ cells than progressing individuals with similar viral loads [3]. Here, we examined whether specific functional properties of the accessory viral Nef protein may contribute to the low levels of $\mathrm{T}$ cell activation and the lack of disease progression in VNPs. Nef is a multi-functional manipulator of the viral host cell that facilitates viral immune evasion and is critical for efficient viral replication and disease progression in HIV-1-infected individuals $[4,5]$. Primate lentiviral Nef proteins generally down-modulate CD4 and MHC-I from the cell surface and enhance viral infectivity and replication $[4,5]$. They differ fundamentally, however, in their effect on the responsiveness of virally infected $\mathrm{T}$ cells to stimulation. HIV-1 Nef proteins may render infected $\mathrm{T}$ cells hyper-responsive to stimulation and promote the induction of cellular transcription factors, 
activation markers and inflammatory cytokines [6-9]. In contrast, most primate lentiviral Nefs block $\mathrm{T}$ cell activation by down-modulation of TCR-CD3 and CD28 from the cell surface [10]. These latter Nef functions are highly conserved in sooty mangabeys that are naturally infected with SIVsmm [11] and may play a protective role in vivo since efficient modulation of TCR-CD3 and CD28 correlates with high and stable $\mathrm{CD} 4^{+} \mathrm{T}$ cell counts in natural SIVsmm infection [12] and in viremic HIV-2-infected individuals [13].

To examine their functional properties, we PCR-amplified nef genes from three previously described HIV-1-infected VNPs from the Amsterdam Cohort Studies on HIV infection and AIDS [3] obtained after six to 14 years of documented HIV-1 infection. For comparison, we utilized nef alleles amplified from plasma samples obtained from eight individuals with progressing HIV-1 infection (P) and seven previously characterized HIV-1, HIV-2, SIVmac and SIVsmm nef alleles (Additional file 1: Table S1). It has previously been shown that SIV Nefs modulate various receptors and enhance viral infectivity and replication in a species-independent manner $[10,14]$. Between four and ten nef alleles were sequenced per VNP and P sample and those predicting amino acid sequences that were identical or almost identical to the respective patient and time point-specific consensus sequence and thus most representative were selected for further analysis (Figure 1). All nef alleles encoded and expressed full-length proteins (Additional file 1: Figures S1) and previously defined functional domains were conserved (Figure 1), suggesting that Nef proteins from both groups of HIV-1-infected individuals are functionally active. No specific sequence signatures were observed in VNP-Nefs, although they frequently contained 22Q $(9 / 11)$ and $62 \mathrm{~T}(9 / 11)$, whereas those from progressing individuals contained $22 \mathrm{R}(5 / 8)$ and $62 \mathrm{~A}$ (6/8; numbers indicate positions in the Nef alignment shown in Figure 1). Given the different cohorts from which the VNP and P samples originated, these are likely to be differences in founder sequences.

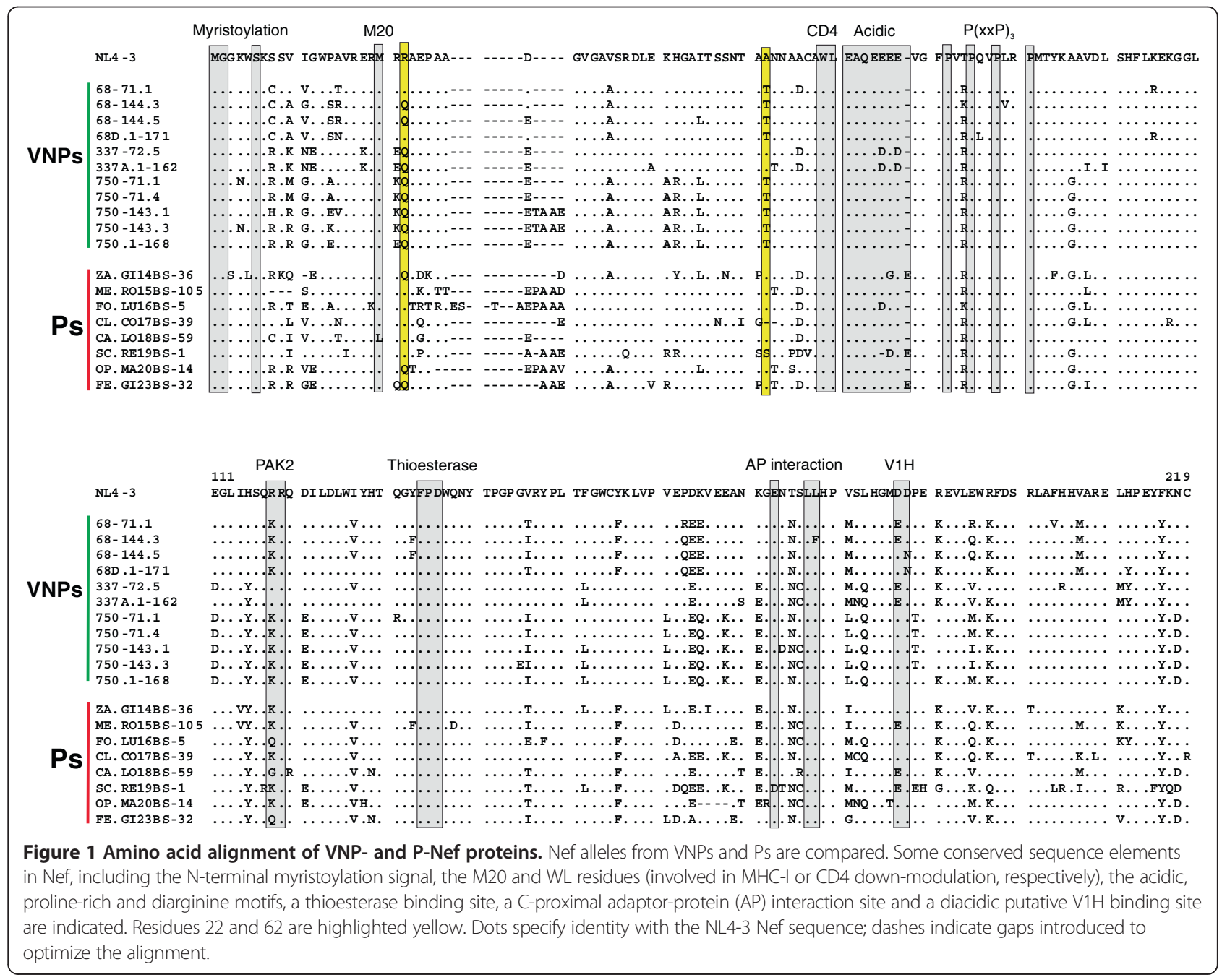


For functional analyses all nef alleles shown in Figure 1 were cloned into an HIV-1 NL4-3 proviral construct that co-expresses Nef and eGFP via an internal ribosomal entry site [14]. Viral particles were generated by cotransfection of 293T cells with the HIV-1 nef-IRES-eGFP proviral constructs and a plasmid (pHIT-G) expressing the vesicular stomatitis virus $G$ glycoprotein and used for transduction of peripheral blood mononuclear cells (PBMCs) or $\mathrm{CD}^{+} \mathrm{T}$ cells as previously described [10,12]. Flow cytometric analyses performed at two days posttransduction showed that nef alleles from both VNPs and Ps down-modulated CD4 and (to a lesser extent) MHC-I and CD28 from the cell surface (Figures 2A-C and Additional file 1: Figure S2). VNP and P Nefs did not show significant differences in receptor modulation but were significantly less active in down-modulating CD28 than HIV-2 and SIV Nefs (Co2) (Figure 2C). Moreover, none of the patient-derived and control HIV-1 Nefs was capable of down-modulating TCR-CD3, whereas HIV-2 and SIV Nefs were highly effective (Figure 2D).

In addition to reducing the lysis of HIV-1-infected cells by cytotoxic $\mathrm{T}$ lymphocytes (CTL) by removing $\mathrm{MHC}-\mathrm{I}$ from the cell surface, Nef is also able to impair CTL function directly by down-modulation of CD $8 \beta[15,16]$. Analyses performed in CEM cells stably expressing A2-CD8 $\beta$ fusions [15] confirmed that CD8 $\beta$ down- modulation is conserved between nef alleles derived from both groups of HIV-1-infected individuals (Figures $2 \mathrm{E}$ and Additional file 1: FigureS2). Unexpectedly, HIV-2, SIVsmm and SIVmac Nefs that all belong to the same lineage of primate lentiviruses were significantly more active than the HIV-1 control Nefs (Figure 2E). Nef may also impair MHC-II-dependent antigen-presentation by up-regulating surface expression of the invariant chain (CD74) [17,18]. Potentially, inefficient activation of $\mathrm{T}$ cells by antigen-presenting cells (APCs) due to potent up-modulation of CD74 may reduce the levels of immune activation. We found, however, that VNP- and P-Nefs did not differ significantly in their ability to enhance CD74 cell surface expression in infected THP-1 cells (Figure 2F). Up-modulation of CD74 was reduced for nef alleles obtained later during infection of VNPs 68 and 337, whereas the ability of VNP-Nefs to modulate other receptors did not change significantly throughout the course of infection (Additional file 1: Figure S3). Whether or not the ability of Nef to suppress MHC-II antigen presentation by upregulation of $\mathrm{CD} 74$ may be modulated throughout the course of HIV-1 infection and if this Nef function plays a role in the pathogenesis of AIDS remains to be analyzed in larger patient cohorts.

The above mentioned results demonstrated that the low levels of immune activation in VNPs were not
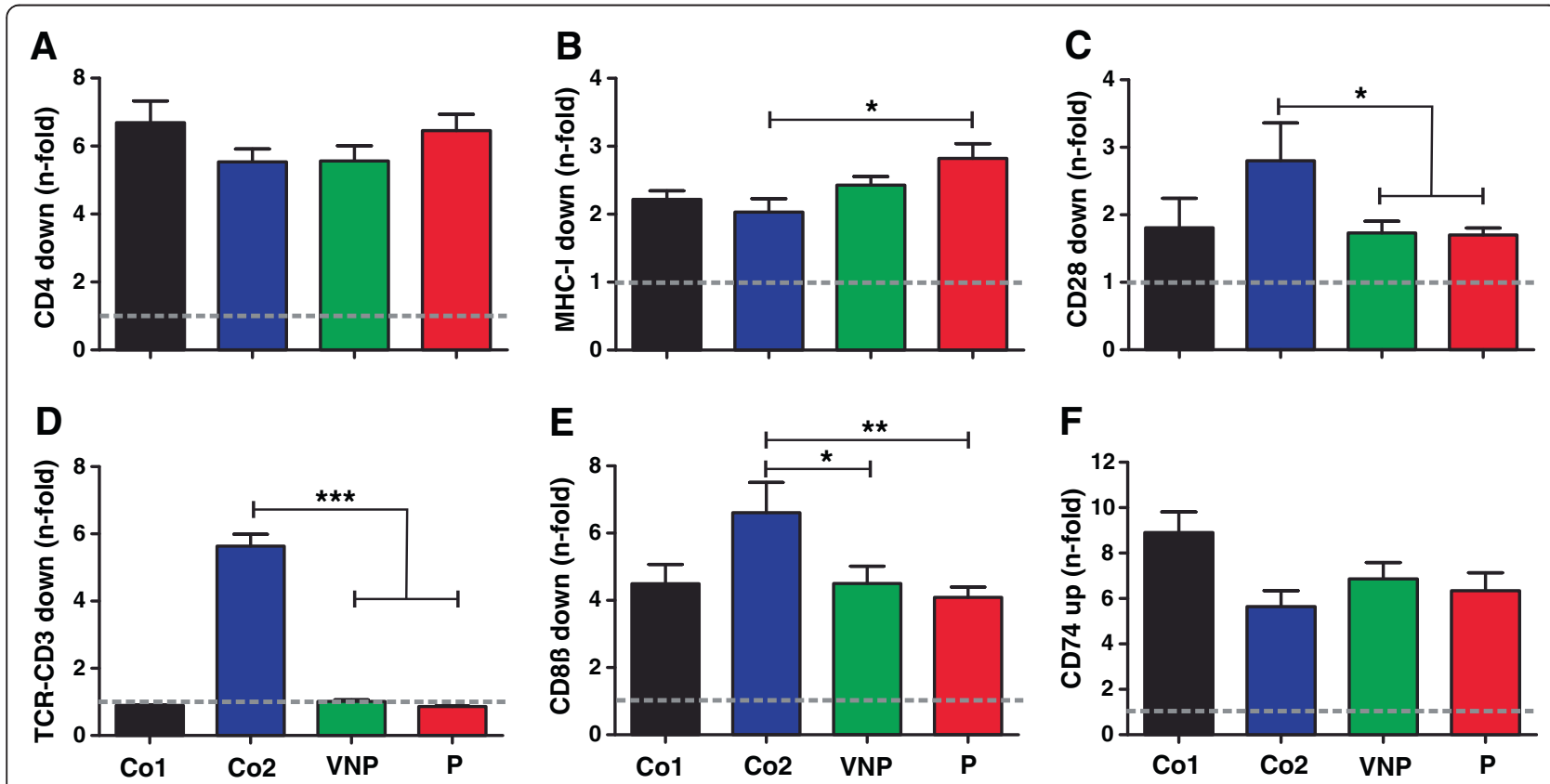

Figure 2 No group-specific difference of Nef-mediated regulation of surface receptors. (A-F) Quantitative assessment of flow cytometric analysis of Nef-mediated modulation of (A) CD4 and (B) MHC-1 in primary CD4 ${ }^{+} T$ cells, (C) CD28 and (D) TCR-CD3 in PBMCs, (E) CD8 $\beta$ in CEM A2-CD8ß fusion cells and (F) CD74 (li) in THP-1 cells transduced with HIV-1 recombinants expressing eGFP alone (nef-) or together with control or patient-derived nef alleles. A vpu and env defective HIV-1 backbone was used to measure the effect of Nef on CD4 surface expression. Given are $n$-fold modulation of cell surface expression compared to the nef-defective control HIV-1 construct (average values \pm standard errors) derived from three independent experiments. Nef alleles were derived from the HIV-1 NL4-3, NA7 and JR-CSF molecular clones (Co 1, black); SIVmac239, HIV-2 BEN, HIV-2 60415 K, SIVsmm FWr1 and FFm1 Nefs (Co2, blue), VNPs ( $n=11$, green) and Ps $\left(n=8\right.$, red). Co, control; ${ }^{*}, p<0.05 ;{ }^{* *}, p<0.01 ;{ }^{* * *}, p<0.001$. 
associated with an increased activity of Nef to modulate receptors involved in the activation of T cells by APCs. It is known, however, that the HIV-1 Nef may also affect $\mathrm{T}$ cell activation by modulating downstream signaling pathways $[19,20]$. To directly examine the effects of Nef on the responsiveness of primary human cells to activation, we infected PBMCs with various HIV-1 IRES/eGFP constructs and stimulated them by PHA treatment as described previously [10]. The results showed that virally infected cells expressing VNP- and P-Nefs expressed higher surface levels of CD69 and CD25 than cells producing HIV-2 or SIV Nefs (Figure 3A, B). Expression of the early and late $\mathrm{T}$ cell activation markers correlated with one another (Figure $3 \mathrm{C}$ ) and inversely with the efficiency of Nef-mediated modulation of CD28 (Figure 3D). Similarly, the levels of apoptosis were higher in HIV-1-infected
PBMCs expressing VNP- and P-Nefs, compared to those expressing SIV or HIV-2 Nefs (Figure 3E) and correlated directly with the expression of $\mathrm{T}$ cell activation markers and inversely (albeit imperfectly) with CD28 cell surface expression (Figure 3F-H). These results are in agreement with previous studies showing that Nef-mediated downmodulation of TCR-CD3 and (to a lesser extent) CD28 suppresses the responsiveness of virally infected $\mathrm{T}$ cells to stimulation and activation-induced cell death $[10,12,13]$.

It has been reported that HIV-1 Nef may increase activation of the nuclear factors of activated T cells and kappa B (NF-AT and NF-kB), respectively [9]. Both of these transcription factors play key roles in innate immunity and inflammatory responses [22,23] and may thus affect the levels of infection-associated immune activation. Measurements of the impact of Nef on NF-AT-dependent

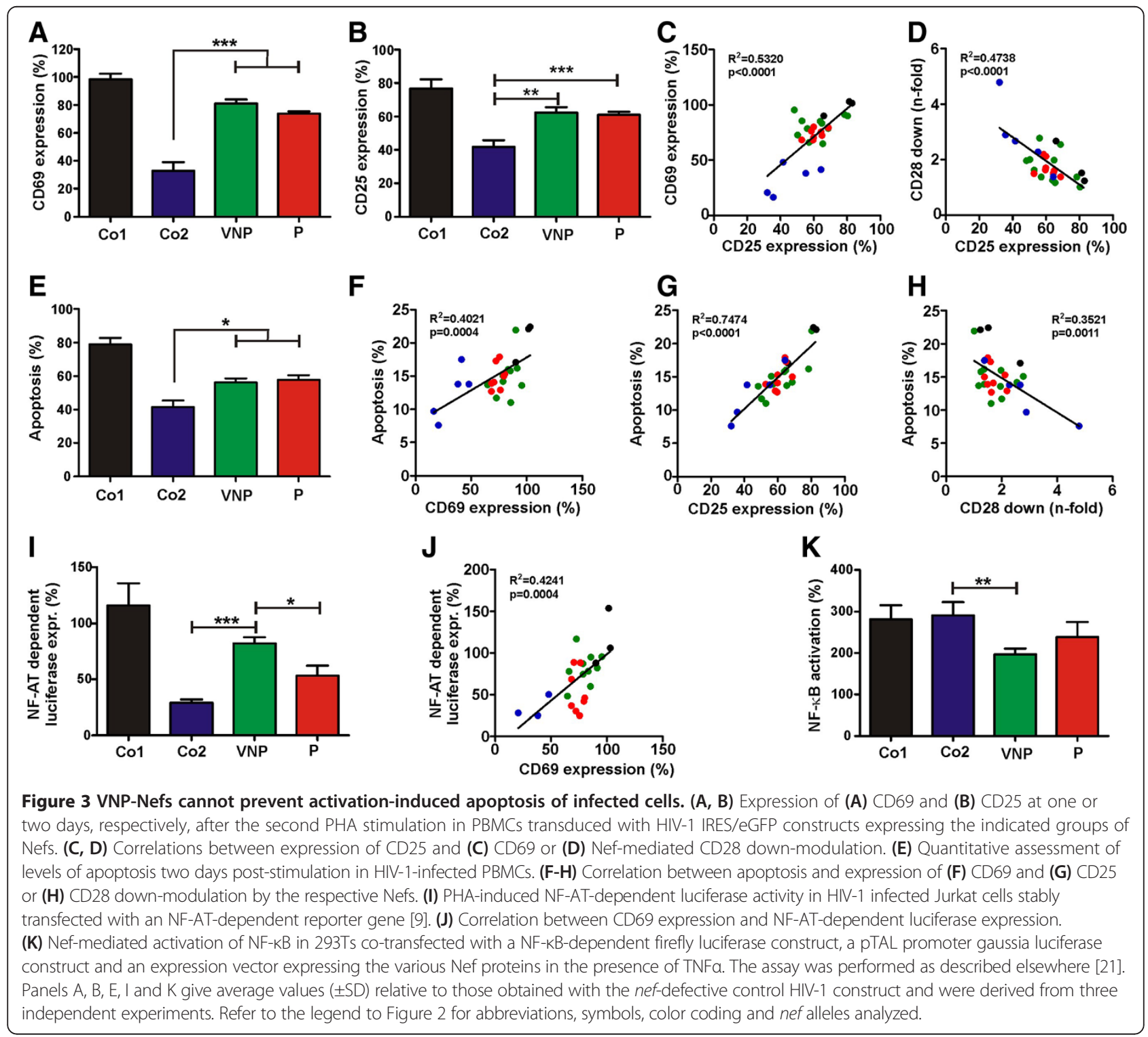


luciferase expression in stably transfected Jurkat $\mathrm{T}$ cells [9] showed that HIV-1 Nefs were generally associated with higher levels of NF-AT activation than HIV-2 and SIV Nefs (Figure 3I, J). While this was expected from published data [9], it came as a surprise that P-Nefs suppressed NF-AT activation more efficiently than VNPNefs. In contrast, P-Nefs trended towards association with higher levels of NF- $\mathrm{BB}$ activation than VNP-Nefs, although this difference failed to reach significance (Figure 3K). Notably, we observed that nef alleles from

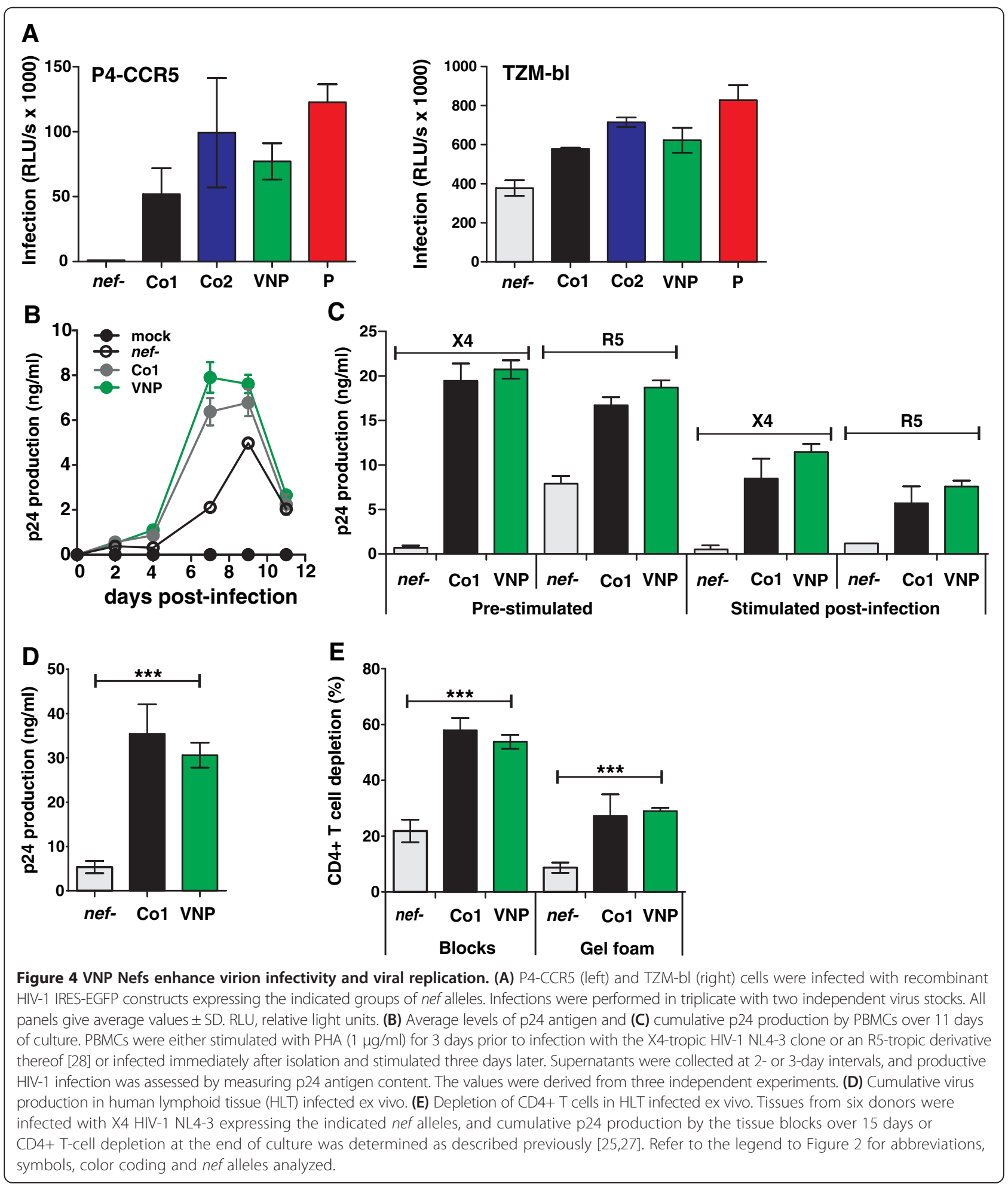


the three $\mathrm{T}$ cell line adapted molecular clones of HIV-1 were associated with higher levels of CD69 ( $p=0.0013)$ and CD25 $(p=0.0012)$ expression, apoptosis $(p=0.0002)$ and NF-AT activation $(\mathrm{p}=0.0111)$ than primary patientderived nef genes (Figure 3). It is also noteworthy that HIV-2 and SIV Nefs efficiently suppressed induction of NF-AT but were particularly active in stimulating NF- $\mathrm{kB}$ (Figure 3I, K). Thus, these primate lentiviruses may efficiently activate the viral LTR via its NF- $\mathrm{kB}$ containing core enhancer element but avoid NF-AT dependent induction of immune response genes.

Finally, we examined the ability of VNP-Nefs to enhance HIV-1 infection, replication and cytopathicity. Infection of P4-CCR5 and TZM-bl indicator cells [24] with virus stocks containing normalized quantities of p24 antigen derived from $293 \mathrm{~T}$ cells transiently transfected with the different proviral constructs [24], showed that most VNPand P-Nefs enhanced virion infectivity, albeit with variable efficacy (Figure 4A, Additional file 1: Figure S3). In agreement with published data [25] the magnitude of the Nef effect on HIV-1 infection was much higher in P4-CCR5 cells than in TZM-bl cells that are highly susceptible to infection. To determine the effect of Nef on HIV-1 replication in primary human cells, PBMCs were infected with HIV-1 constructs and virus production in the supernatant was determined as described previously [26]. In agreement with the high viral loads, VNP-Nefs increased virus production (Figure 4B), irrespectively of the viral coreceptor tropism, and of whether or not the PBMCs were pre-stimulated or stimulated 3 days after infection (Figure 4C). Since PBMC cultures may not faithfully recapitulate the viral phenotype in vivo, we also determined the levels of viral replication and $\mathrm{CD} 4^{+} \mathrm{T}$ cell depletion in ex vivo human tonsillary tissues that allow determination of the cytopathicity and replication capacity of HIV-1 without exogenous stimulation [27]. Since the number of cultures that could be set up per tonsil was limited, we only compared the effect of the VNP-Nefs with the HIV-1 control Nefs. We found that HIV-1 constructs expressing VNP-Nefs replicated efficiently in human lymphoid tissue (HLT) and depleted $\mathrm{CD} 4^{+} \mathrm{T}$ cells as effectively as otherwise isogenic viral strains expressing the control NL4-3 and NA7 Nefs (Figure 4D, E). These results are in agreement with the previous finding that biologically cloned HIV-1 isolates from these three VNPs are cytopathic and replication competent in ex vivo infected human lymphoid tissue [3].

In conclusion, low levels of chronic inflammation and lack of disease progression in VNPs are not due to efficient Nef-mediated suppression of $\mathrm{T}$ cell activation. The possible relevance and significance of the modest differences in modulation of NF-AT and NF-kB by VNP- and P-Nefs observed in the present study needs to be further examined in larger patient cohorts. Notably, our data suggest that nef alleles derived from primary HIV-1 strains but not those of $\mathrm{T}$ cell line adapted molecular clones of HIV-1 may suppress $\mathrm{T}$ cell activation, albeit much less efficiently than HIV-2 and SIV nef genes that down-modulate TCR-CD3. Thus, our results further underline the necessity to use primary nef alleles to avoid in vitro artifacts. Finally, our findings suggest that host factors rather than specific viral properties may allow VNPs to avoid harmful chronic immune activation by HIV-1 replication.

\section{Additional file}

\begin{abstract}
Additional file 1: Overview on nef alleles analyzed. Figure S1.
Expression of Nef proteins. Western blot analysis of lysates from 293T cells transfected with pCGCG vectors expressing AU-1-tagged versions of the indicated Nef proteins. Lysates were probed with an anti AU-1 monoclonal antibody (Covance), anti $\beta$-actin polyclonal antibody (Abcam) and anti GFP polyclonal antibody (Abcam). Figure $\mathbf{S 2}$. Modulation of various receptors by VNP- and P-Nefs. Primary CD4+ T cells were transduced with NL4-3 constructs coexpressing the indicated nef alleles and GFP and assayed by FACS. CEM cells expressing A2-CD8 $\beta$ fusions and THP-1 cells were utilized to examine modulation of $C D 8 ß$ and CD74 expression, respectively. The ranges of eGFP expression used to calculate receptor modulation in Figure 1 are indicated. Figure S3. Activity of VNP-Nefs throughout the course of infection. (A-F) Quantitative assessment of Nef-mediated modulation of (A) CD4, (B) $\mathrm{MHC}-\mathrm{I},(\mathrm{C}) \mathrm{CD} 28$ and (D) TCR-CD3 in primary cells, (E) CD8 $\beta$ in CEM A2-CD8 $\beta$ fusion cells and (F) CD74 (li) in THP-1 cells transduced with HIV-1 recombinants expressing eGFP alone (nef-) or together with various nef alleles. (G to I) Quantitative analysis of (G) CD69 expression, (H) CD25 expression or (I) apoptosis levels in transduced PBMCs. (J) PHA-induced NF-AT-dependent luciferase activity obtained from transduced Jurkat cells stably transfected with an NF-AT-dependent reporter gene. (K) Analysis of Nef-mediated activation of NF-KB in 293Ts co-transfected with a NF-KB-dependent firefly luciferase construct, a pTAL promoter gaussia luciferase construct (to normalize) and Nef expression vectors in the presence of TNFa. (L) Nef-mediated enhancement of infectivity in P4-CCR5 cells. Given are average values \pm SEM derived from multiple experiments of Nefs from HIV-1 NA7 (white), HIV-2 BEN (blue), VNP 68 (light green), VNP 337 (middle green) and VNP 750 (dark green). Numbers below bars provide month of sampling after the estimated data of primary infection.
\end{abstract}

\section{Competing interests}

The authors declare no conflict of interest.

\section{Authors' contributions}

$\mathrm{AH}$ performed most experiments. DC and AvW contributed reagents. $\mathrm{AH}$ and FK designed the study, performed the statistical analysis, and wrote the manuscript. All authors read and approved the final manuscript.

\section{Acknowledgements}

The authors thank Kerstin Regensburger, Martha Meyer, Birgit Ott and Daniela Krnavek for excellent technical assistance, Dre van der Merwe and Daniel Sauter for critical reading, and Heinz Maier from the Military Hospital Ulm and Thomas Hoffmann from the Department of Ear-Nose and Throat, Ulm University Medical Center for providing tonsillary tissues. Further we thank Bernd Baumann, University UIm for providing the NF-kB firefly luciferase reporter plasmid. HIV-infected progressor ( $P$ ) samples were sampled from the BRESCIA cohorts and kindly provided by Carlo Torti. This work was supported by the Deutsche Forschungsgemeinschaft (DFG) and the European Research Council (ERC).

The Amsterdam Cohort Studies on HIV infection and AIDS, a collaboration between the Amsterdam Health Service, the Academic Medical Center of the University of Amsterdam, Sanquin Blood Supply Foundation, the University Medical Center Utrecht, and the Jan van Goyen Clinic are part of the Netherlands HIV Monitoring Foundation and financially supported by the 
Center for Infectious Disease Control of the Netherlands National Institute for Public Health and the Environment. All experiments reported in this study and bllod sampling were performed with the approval of the local ethics committees. We are indebted to all participants for their continuous participation in the study.

\section{Author details}

IInstitute of Molecular Virology, Ulm University Medical Center, Ulm, Germany. ${ }^{2}$ Cancer Research Institute, Institute for Immunology, Center for Virus Research, University of California Irvine, Irvine, California, USA. ${ }^{3}$ Department of Experimental Immunology, Sanquin Research, Landsteiner Laboratory and Center for Infection and Immunity Amsterdam (CINIMA), Academic Medical Center of the University of Amsterdam, Amsterdam, The Netherlands. ${ }^{4}$ Present address: Crucell Holland BV, Leiden, The Netherlands.

Received: 1 October 2013 Accepted: 26 January 2014

Published: 4 February 2014

\section{References}

1. Sodora DL, Allan JS, Apetrei C, Brenchley JM, Douek DC, Else JG, Estes JD, Hahn BH, Hirsch VM, Kaur A, Kirchhoff F, Muller-Trutwin M, Pandrea I, Schmitz JE, Silvestri G: Toward an AIDS vaccine: lessons from natural simian immunodeficiency virus infections of African nonhuman primate hosts. Nat Med 2009, 15:861-865.

2. Chahroudi A, Bosinger SE, Vanderford TH, Paiardini M, Silvestri G: Natural SIV hosts: showing AIDS the door. Science 2012, 335:1188-1193.

3. Choudhary SK, Vrisekoop N, Jansen CA, Otto SA, Schuitemaker H, Miedema F, Camerini D: Low immune activation despite high levels of pathogenic human immunodeficiency virus type 1 results in long-term asymptomatic disease. J Virol 2007, 81:8838-8842.

4. Kirchhoff F: Role of Nef in primate lentiviral immunopathogenesis. Cell Mol Life Sci 2008, 65:2621-2636.

5. Arien KK, Verhasselt B: HIV Nef: role in pathogenesis and viral fitness. Curr HIV Res 2008, 6:200-208.

6. Skowronski J, Parks D, Mariani R: Altered T cell activation and development in transgenic mice expressing the HIV-1 nef gene. EMBO 1993, 12:703-713.

7. Wang JK, Kiyokawa E, Verdin E, Trono D: The Nef protein of HIV-1 associates with rafts and primes T cells for activation. Proc Natl Acad Sci USA 2000, 97:394-399.

8. Fenard D, Yonemoto W, de Noronha C, Cavrois M, Williams SA, Greene WC Nef is physically recruited into the immunological synapse and potentiates T cell activation early after TCR engagement. J Immunol 2005, 175:6050-6057.

9. Fortin JF, Barat C, Beauséjour Y, Barbeau B, Tremblay MJ: Hyper-responsiveness to stimulation of human immunodeficiency virus-infected CD4+ T cells requires Nef and Tat virus gene products and results from higher NFAT, NF-kappaB, and AP-1 induction. J Biol Chem 2004, 279:39520-39531.

10. Schindler M, Münch J, Kutsch O, Li H, Santiago ML, Bibollet-Ruche F, Müller-Trutwin MC, Novembre FJ, Peeters M, Courgnaud V, Bailes E, Roques R, Sodora DL, Silvestri G, Sharp PM, Hahn BH, Kirchhoff F: Nef-mediated suppression of T cell activation was lost in a lentiviral lineage that gave rise to HIV-1. Cell 2006, 125:1055-1067.

11. Schmökel J, Li H, Bailes E, Schindler M, Silvestri G, Hahn BH, Apetrei C, Kirchhoff F: Conservation of Nef function across highly diverse lineages of SIVsmm. Retrovirology 2009, 6:36

12. Schindler M, Schmökel J, Specht A, Li H, Münch J, Khalid M, Sodora DL, Hahn BH, Silvestri G, Kirchhoff F: Inefficient Nef-mediated downmodulation of CD3 and MHC-I correlates with loss of CD4 + T cells in natural SIV infection. PLoS Pathog 2008, 4:e1000107.

13. Khalid M, Yu H, Sauter D, Usmani SM, Schmökel J, Feldman J, Gruters RA van der Ende ME, Geyer M, Rowland-Jones S, Osterhaus AD, Kirchhoff F: Efficient Nef-mediated downmodulation of TCR-CD3 and CD28 is associated with high CD4+ T cell counts in viremic HIV-2 infection. J Virol 2012, 86:4906-4920.

14. Münch J, Rajan D, Schindler M, Specht A, Rücker E, Novembre FJ, Nerrienet E, Müller-Trutwin MC, Peeters M, Hahn BH, Kirchhoff F: Nef-mediated enhancement of virion infectivity and stimulation of viral replication are fundamental properties of primate lentiviruses. J Virol 2007, 81:13852-13864.
15. Leonard JA, Filzen T, Carter CC, Schaefer M, Collins KL: HIV-1 Nef disrupts intracellular trafficking of MHC-I, CD4, CD8, and CD28 by distinct pathways that share common elements. J Virol 2011, 85:6867-6881.

16. Heigele A, Schindler M, Gnanadurai CW, Leonard JA, Collins KL, Kirchhoff F: Down-modulation of CD8a $\beta$ is a fundamental activity of primate lentiviral Nef proteins. J Virol 2012, 86:36-48.

17. Stumptner-Cuvelette P, Morchoisne S, Dugast M, Le Gall S, Raposo G, Schwartz O, Benaroch P: HIV-1 Nef impairs MHC class II antigen presentation and surface expression. Proc Natl Acad Sci U S A 2001, 98:12144-12149.

18. Schindler M, Wildum S, Casartelli N, Doria M, Kirchhoff F: Nef alleles from children with non-progressive HIV-1 infection modulate MHC-II expression more efficiently than those from rapid progressors. AIDS 2007, 21:1103-1107.

19. Renkema GH, Saksela K: Interactions of HIV-1 Nef with cellular signal transducing proteins. Front Biosci 2000, 5:D268-D283.

20. Greenway AL, Holloway G, McPhee DA, Ellis P, Cornall A, Lidman M: HIV-1 nef control of cell signaling molecules: multiple strategies to promote virus replication. J Biosci 2003, 28:323-335.

21. Hotter D, Kirchhoff F, Sauter D: HIV-1 Vpu does not degrade interferon regulatory factor 3. J Virol 2013, 87:7160-7165.

22. Fric J, Zelante T, Wong AY, Mertes A, Yu HB, Ricciardi-Castagnoli P: NFAT control of innate immunity. Blood 2012, 120:1380-1389.

23. Pitha PM: Innate antiviral response: role in HIV-1 infection. Viruses 2011, 3:1179-1203.

24. Charneau PG, Mirambeau P, Roux P, Paulous S, Buc H, Clavel F: HIV-1 reverse transcription. A termination step at the center of the genome. J Mol Biol 1994, 241:651-662

25. Schindler M, Rajan D, Specht A, Ritter C, Pulkkinen K, Saksela K, Kirchhoff F: Association of Nef with p21-activated kinase 2 is dispensable for efficient human immunodeficiency virus type 1 replication and cytopathicity in ex vivo-infected human lymphoid tissue. J Virol 2007, 81:13005-13014.

26. Specht A, Telenti A, Martinez R, Fellay J, Bailes E, Evans DT, Carrington M, Hahn BH, Goldstein DB, Kirchhoff F: Counteraction of HLA-C-mediated immune control of HIV-1 by Nef. J Virol 2010, 84:7300-7311.

27. Glushakova S, Grivel JC, Suryanarayana K, Meylan P, Lifson JD, Desrosiers R, Margolis L: Nef enhances human immunodeficiency virus replication and responsiveness to interleukin-2 in human lymphoid tissue ex vivo. J Virol 1999, 73:3968-3974

28. Papkalla A, Münch J, Otto C, Kirchhoff F: Nef enhances human immunodeficiency virus type 1 infectivity and replication independently of viral coreceptor tropism. J Virol 2002, 76:8455-8459.

doi:10.1186/1742-4690-11-13

Cite this article as: Heigele et al:: Viremic long-term nonprogressive HIV-1 infection is not associated with abnormalities in known Nef functions. Retrovirology 2014 11:13.

\section{Submit your next manuscript to BioMed Central and take full advantage of:}

- Convenient online submission

- Thorough peer review

- No space constraints or color figure charges

- Immediate publication on acceptance

- Inclusion in PubMed, CAS, Scopus and Google Scholar

- Research which is freely available for redistribution 